\begin{tabular}{|c|c|}
\hline $\begin{array}{c}\text { Yanbu Journal } \\
\text { of } \\
\text { Engineering } \\
\text { and Science }\end{array}$ & \\
\hline ISSN: 1658-5321 & $\begin{array}{c}\text { Vol. 16, June } 2018(1439 H) \\
\text { www.yjes.org.sa }\end{array}$ \\
\hline
\end{tabular}

\title{
DEVELOPMENT OF AN OPTIMAL PATH PLANNING USING ELITE OPPOSITION BASED BAT ALGORITHM FOR A MOBILE ROBOT
}

\author{
Zaharudeen Haruna*, Muhammad B. Mu'azu, Prosper Oyibo, Salawuden A. Tijani \\ Dept.of Electrical \& Computer Engineering, Ahmau Bello Univeristy, Zaria, Nigeria \\ E-mail: elzet2007@gmail.com
}

\begin{abstract}
This research work presents the development of an optimal path planning using elite opposition based bat algorithm (EOBA) for mobile robot, such that the robot avoids obstacle(s) without making contact with them. The bat algorithm (BA) is a nature inspired meta-heuristic algorithm that works on the basis of the echolocation behavior of bat. It, however, has a poor exploration capability leading to it easily getting stuck in local optima. The EOBA is developed by modifying the BA with the elite opposition-based learning (EOBL) so as to diversify the solution search space and the inertial weight in order to balance its exploration and exploitation. The performance of the proposed path planning technique was compared with that of the standard BA based on the ability to generate an optimal path for a mobile robot in a developed simulation environment. The simulation results showed that EOBA provide an optimal path with minimum elapsed time as compared to that of the standard BA. All simulations were carried out using MATLAB R2013b.
\end{abstract}

Keywords: Bat Algorithm, EOBA, Optimal path, Path planning.

\section{INTRODUCTION}

The field of robot path planning was launched in the middle of the 1960's. Robot path planning is an essential problem in navigation of mobile robots. The aim is to find an optimal and collisionfree path from a predefined start position to a target point in a given environment. Generally, there are many paths for a robot to reach its target, but the best path is selected according to some guidelines. These guidelines include: shortest distance, least energy consuming or shortest time with the shortest distance [1]. So, for designing a fast and efficient procedure for navigation, path planning is an essential aspect. The path planning problem consists of finding a sequence of moves for rearranging the robot in a certain environment, from a certain initial position in the environment to the given goal position, and the robot must avoid obstacles in the environment. The major challenges for robot path-planning problems are computational complexity, local optimum and adaptability [2]. Mobile robot path planning is divided into global path planning and local path planning. The global path planning (off-line) of robots is done in an environment where complete information about stationary obstacles and trajectory of moving obstacles are known in advance, so that the robot only needs to compute the path once at the beginning and then to follow the planned path up to the target point. When complete information about environment is not available in advance, mobile robot gets information through sensors as it moves 
through the environment, this is known as on-line or local path planning [3]. For robots to be able to execute various types of tasks with minimum or no human intervention, robots need to be more autonomous and intelligent [4]. Autonomous mobile robots have many applications in areas such as material transportation, hazardous environment, military operation, mines excavations, computer animation, drug design and automated surveillance. During the last two decades, researchers have proposed many techniques for control and automation. Such techniques include, proportional-integral-differential (PID) control, fuzzy logic control, neural network, metaheuristic search algorithm and hybrid. For robotic path planning, metaheuristic search algorithm is better technique because it is problem independent, and it uses random solutions to achieve its goal. Most of these metaheuristic algorithms are inspired, mimicking the successful features of the underlying biological, physical or sociological systems. The bat algorithm is a metaheuristic search algorithm inspired by the echolocation characteristic of bats [5]. The primary purpose for bat's echolocation is to serve as a hunting strategy. However, the bat algorithm has a high rate of exploitation, but at times it may get trapped into some local optima, so that it may not perform exploration very well [6]. As such, when applied in robotic path planning to generate an optimal path, the robot always make contact with the obstacle before avoiding it. This was the main reason for the development of the elite opposition based bat algorithm (EOBA).

\section{THE BAT ALGORITHM}

Xin-She Yang in 2010 developed a new nature inspired algorithm known as Bat algorithm, which was based on the echolocation characteristics of micro bats
[5]. The algorithm exploits the so-called echolocation of the bats. Echolocation is typical sonar which bats use to detect prey and to avoid obstacles. It is generally known that sound pulses are transformed into frequency which reflects from obstacles. The bats navigate by using the time delay from emission to reflection. The pulse rate can be simply determined in the range from 0 to 1 , where 0 means that there is no emission and 1 means that the bat's emitting is at their maximum. In order to transform these behaviors of bats to algorithm, Yang used three idealized rules [5]:

1. All bats use echolocation to sense distance, and they also know the difference between food/prey and background barriers;

2. Bats fly randomly with velocity $v_{i}$ at position $x_{i}$ with a fixed frequency $\left(Q_{\text {min }}\right)$, varying wavelength $\lambda$ and loudness $A_{0}$ to search for prey. They automatically adjust the wavelength (or frequency) of their emitted pulses and adjust the rate of pulse emission $r \in[0,1]$, depending on the proximity of the target;

3. The loudness can vary from a large (positive) $A_{0}$ when searching for prey to a minimum constant value $\left(A_{\min }\right)$ when homing towards the prey.

The basic steps of implementing Bat algorithm are described as follows [5]:

\subsection{MOVEMENT OF VIRTUAL BATS}

Just like the real bats, the virtual bats fly randomly by adjusting its frequency $\mathrm{Q}$ first and updating its velocity $v_{i}$ and position $x_{i}$ next. The new locations/solutions $x_{i}^{t}$ and velocities $v_{i}^{t}$ at time step $t$ are represented in [5]:

$$
Q_{i}=Q_{\min }+\left(\mathrm{Q}_{\max }-Q_{\min }\right) U(0,1)
$$




$$
\begin{aligned}
& v_{i}^{t}=v_{i}^{t-1}+\left(x_{i}^{t}-x_{\text {best }}\right) Q_{i} \\
& x_{i}^{t}=x_{i}^{t-1}+v_{i}^{t}
\end{aligned}
$$

where $\mathrm{U}(0,1)$ is used for random distribution, $x_{\text {best }}$ is used for current global best location (solution) that is calculated by comparing all the solutions among all the bats [5]. The current best solution for each bat is generated locally using random walk as given in [5]:

$$
x_{\text {new }}=x_{\text {old }}+\varepsilon A^{t}
$$

where $\varepsilon \in[-1,1]$ is a random number between -1 to 1 , and $A^{t}$ is used for average loudness of all the bats at time step $t[5]$.

\subsection{LOUDNESS AND PULSE EMISSION}

The general rule is that the loudness decreases while the rate of pulse emission increases, when a bat has found its prey. The loudness and pulse emission rate are updated by the following equations [5]:

$$
\begin{aligned}
& A_{i}^{t+1}=\alpha A_{i}^{t} \\
& r_{i}^{t+1}=r_{i}^{0}[1-\exp (-\gamma t)]
\end{aligned}
$$

where $A_{i}^{t}$ and $A_{i}^{t+1}$ are the previous and updated values of the loudness for the $i^{\text {th }}$ bat, $\alpha$ and $\gamma$ are constants, and $r_{i}^{t+1}$ is the pulse rate of the $i^{\text {th }}$ bat at iteration $t+1$. For any $0<\alpha<1$ and $\gamma>0$, [5]

$$
A_{i}^{t} \rightarrow 0, r_{i}^{t} \rightarrow r_{i}^{0}, \text { as } t \rightarrow \infty \text {. }
$$

\section{ELITE OPPOSITION BASED BAT ALGORITHM}

Elite opposition based bat algorithm is developed by introducing elite oppositionbased learning at the initialization of the standard bat algorithm in order to diversify the solution search space so that the algorithm can avoid being trapped in local optima. At the same time, inertial weight was introduced to the local search part of the algorithm in order to improve the exploitation capability of the algorithm so as to establish balance between exploration and exploitation during the search process of the algorithm.

\subsection{ELITE OPPOSITION BASED LEARNING}

Tizhoosh [7] introduced opposition-based learning (OBL) as a method to accelerate the performance of various computational intelligence techniques by increasing the diversity in the population so as to explore different regions that exist in the solution domain [8]. In the context of OBL, generation jumping is used to control the probability of triggering the opposite operation, and it can be considered as a pattern of mutation for encouraging diversity to some extent. In general, the generation jumping rate is set to a relative small value, therefore how to generate more promising opposite individuals seems to be important [9]. EOBL is a new technique in the field of intelligence computation. Its main premise is to utilize some selected elite individuals from the current population to generate a corresponding opposite population located within the dynamic search boundaries by opposition-based learning. The search space is then guided to approximate the promising regions in which the global optimum may be included by simultaneously evaluating the current population and the opposite one. This approach is helpful in taking advantage of the best characteristics of the elite individuals and encouraging diversity for avoidance of premature convergence [9]. Along with the aforementioned idea of improving OBL, some elite individuals from the current population are utilized in EOBL instead of the ordinary individuals in the case of the OBL. From preliminary experiments, it is observed that the opposite solution of an elite individual is more likely to be located in a region in which the global optimum is expected to be located [9]. 
Suppose that the elite individual of the population is $x_{e}=\left(x_{e, 1}, x_{e, 2}, \ldots, x_{e, D}\right)$, for the individual $x_{i, j}=\left(x_{i, 1}, x_{i, 2}, \ldots, x_{i, D}\right)$, the elite opposition-based solution $\tilde{x}_{i, j}$ can be obtained by the following equation [10]:

$$
\tilde{x_{i, j}}=\delta\left(a_{j}+b_{j}\right)-x_{e, j}
$$

where $i=1,2, \ldots, n$ and $j=1,2, \ldots, D, \mathrm{n}$ is the population size, $\mathrm{D}$ is the dimension of $x$, $\delta \in U(0,1), \quad\left(a_{j}, b_{j}\right)$ is the dynamic decision bound of the $j^{\text {th }}$ decision variable and can be calculated as:

$$
a_{j}=\min \left(x_{i, j}\right) \text { and } \mathrm{b}_{j}=\max \left(x_{i, j}\right)
$$

The use of dynamic boundary instead of fixed boundary can help to save search experience and make opposite solution lie in a search space which is reduced gradually [11].

\subsection{INERTIA WEIGHT FACTOR}

In order to improve the exploitation capability of the bat algorithm, inertia weight is introduced in the local search stage of the standard bat algorithm. The introduction of the inertia weight is to keep track of the previous history of the best solution at the local search stage of the algorithm, thus ensuring that the search region of the best solution always minimizes as the algorithm moves towards the termination criteria. The inertia weight that will be chosen for the purpose of this research is a linear value of iteration weight. The choice of this inertia weight is to ensure that the solution search space always minimizes as the algorithm moves towards the termination criteria during exploitation. This is given in equation (10) [12]:

$$
\lambda=\left(\frac{i t r_{\max }-i t r}{i t r_{\max }}\right)^{n}
$$

Where $i t r_{\max }$ correspond to the maximum iteration number and itr correspond to the current iteration number. This weight in equation (10) is then utilized into the local search equation of the standard bat algorithm as shown below:

$$
x_{\text {new }}=x_{\text {old }}+\varepsilon \lambda A^{t}
$$

Equation (11) is expected to make significant improvement to the convergence accuracy of the proposed modified algorithm as the exploitation capability of the algorithm is improved. This will enable the algorithm obtain an optimum or near optimum result. The flow chart of the EOBA is given in Figure 1.

\section{OPTIMAL PATH PLANNING}

The path planning problem is to determine a path of a mobile robot from its initial point called source location to a destination point called target location in an environment populated with obstacles. The desired path is optimal if the mobile robot navigates to the target in a minimum time (i.e. shortest distance) without colliding with the obstacles in the environment. Suppose a robot starts its movement from a source location $\left(X_{1}, Y_{1}\right)$ represented by a red square with coordinate $(10,0)$ to a target location $\left(X_{2}, Y_{2}\right)$ represented by a green diamond with coordinate $(120,120)$ in a $120 * 120$ environment containing 20 obstacles as shown in Figure 2. 


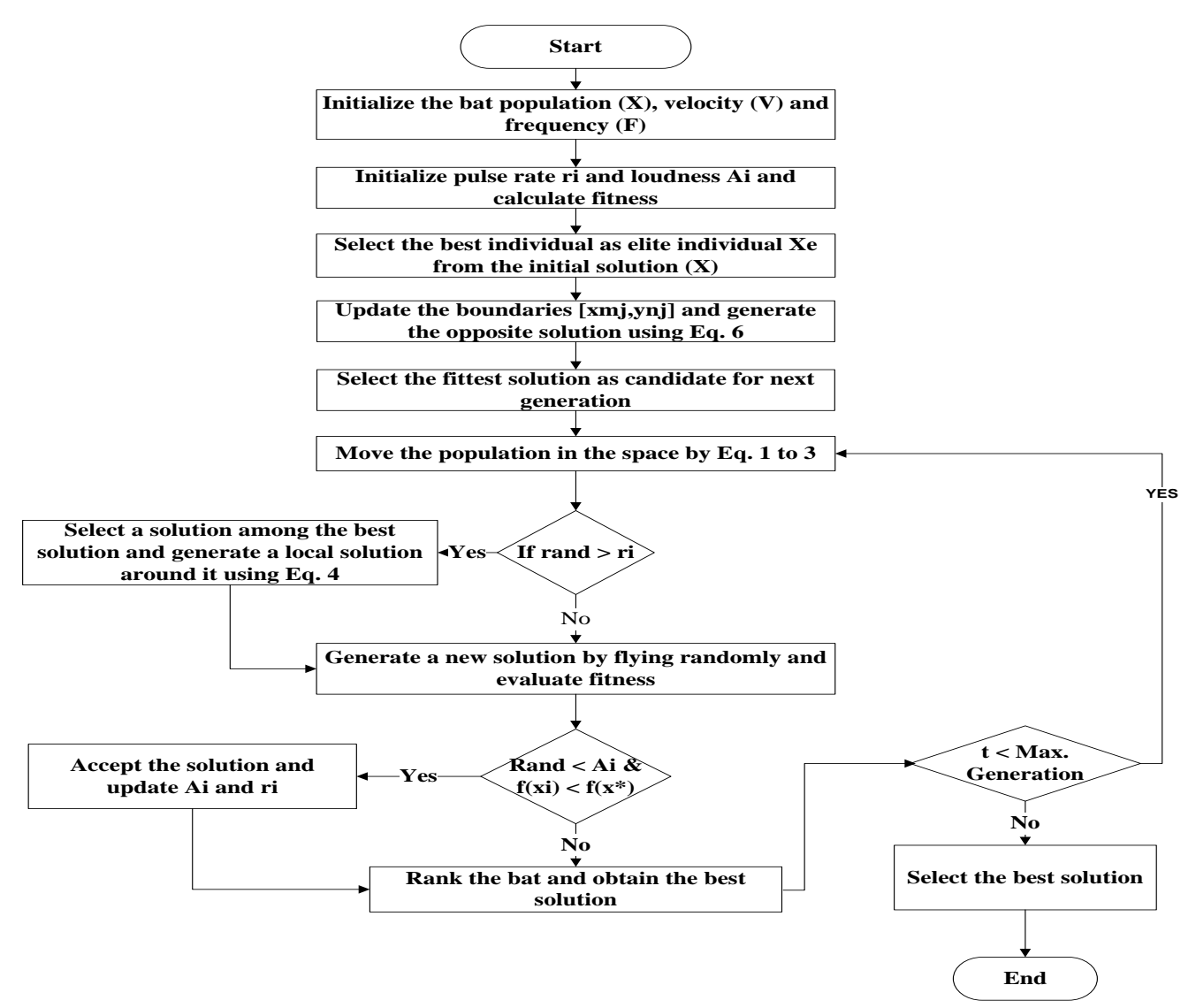

Fig. 1 Flow Chart of the Modified Bat Algorithm

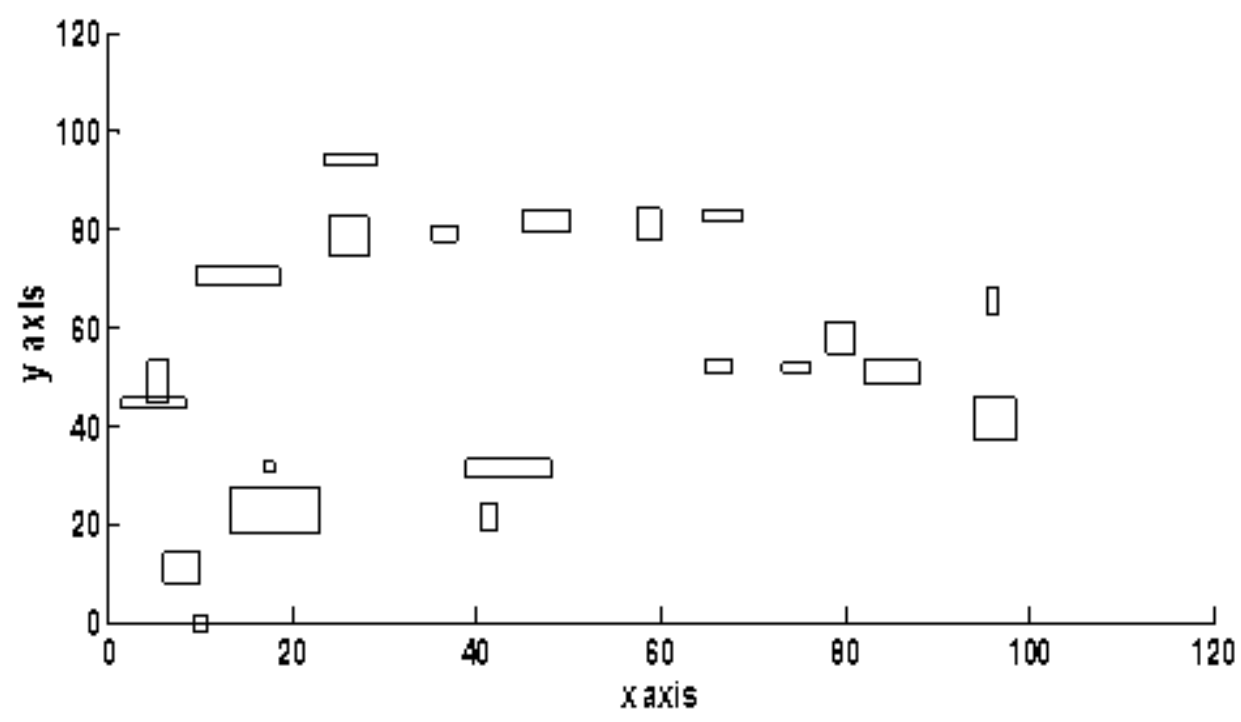

Fig. 2 Path Planning Simulation Environment 


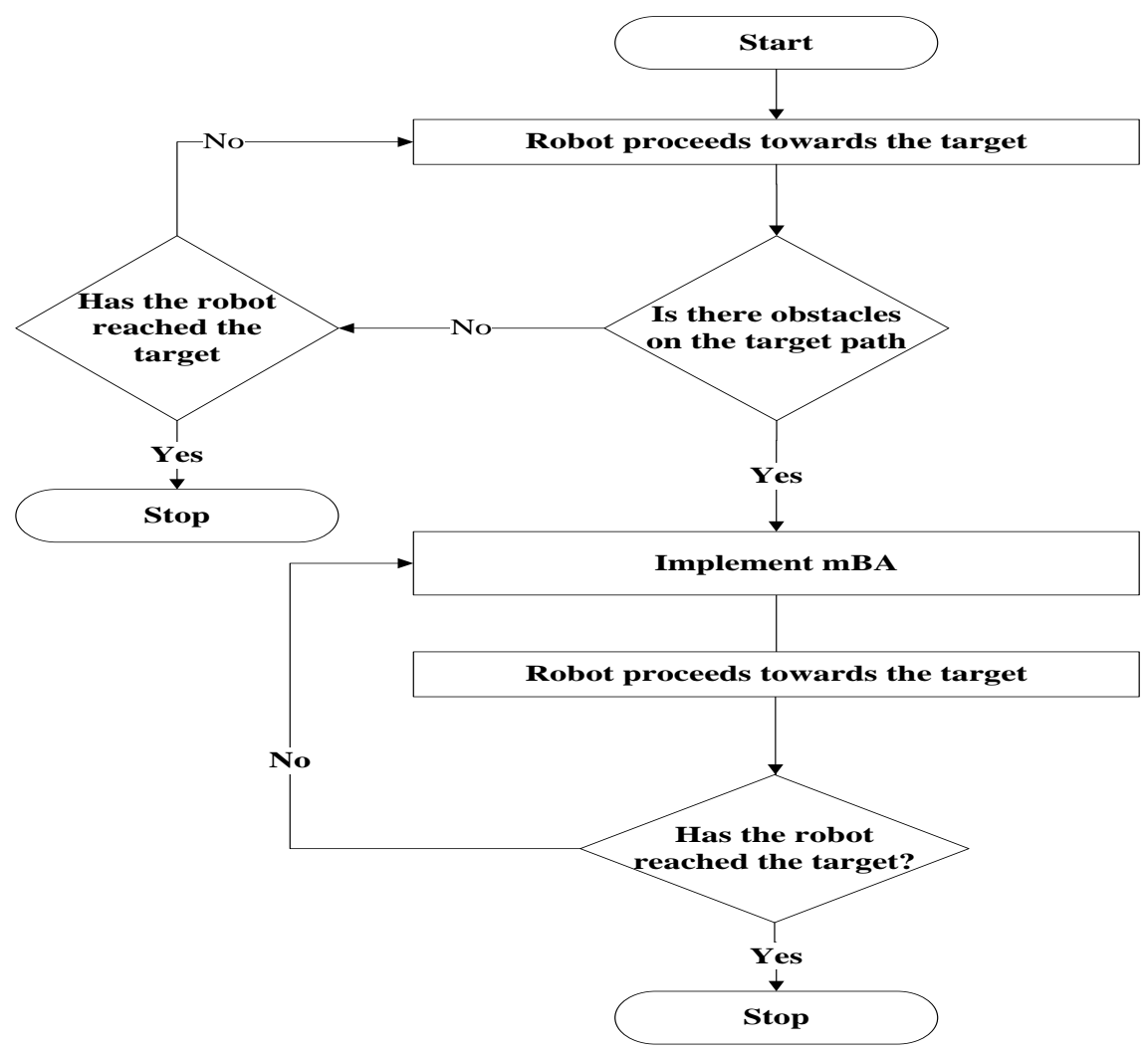

Fig. 3 Flow Chart of Robot Path Planning using EOBA

The distance between the source location and the target location is calculated by the following distance formula:

$$
d=\sqrt{\left(X_{2}-X_{1}\right)^{2}+\left(Y_{2}-Y_{1}\right)^{2}}
$$

The direction of movement $\theta$, is determine using equation (13):

$$
\theta=\tan ^{-1}\left(\frac{a b s\left(Y_{2}-Y_{1}\right)}{a b s\left(X_{2}-X_{1}\right)}\right)
$$

As the robot move one step ahead, the value of $X_{1}$ and $Y_{1}$ are updated based on equations (13) and (14) respectively:

$$
\begin{aligned}
& X_{1}=X_{1}+\text { step }^{*} \cos (\theta) \\
& Y_{1}=Y_{1}+\text { step }^{*} \sin (\theta) \\
& \text { where } \text { step }=1
\end{aligned}
$$

The nearest obstacle to the robot can be detected using equation (16):

$$
d_{R O B-O B S T}=\sqrt{\left(X_{O B S T}-X_{R O B}\right)^{2}+\left(Y_{O B S T}-Y_{R O B}\right)^{2}}
$$

where $\left(X_{\text {OBST }}, Y_{O B S T}\right)$ is the coordinate of the obstacle and $\left(X_{R O B}, Y_{R O B}\right)$ is the coordinate of the robot. If the robot does not detect any obstacle(s) in its target path during the search for the optimal path, then it will travel towards its target in a relatively straightforward manner using equation (14) and (15). But if the robot detects an obstacle along its path based on equation (16), the EOBA path planning algorithm is utilized to steer the robot within the environment to avoid them. The developed flow chart diagram for the robot path planning using EOBA is given in Figure 3. As the mobile robot moves from the source location to the target location without encountering any obstacle during its search for optimal path, the robot continues to move until the target location is reached as 
described in Figure 3. The simulation result is shown in Figure 4.

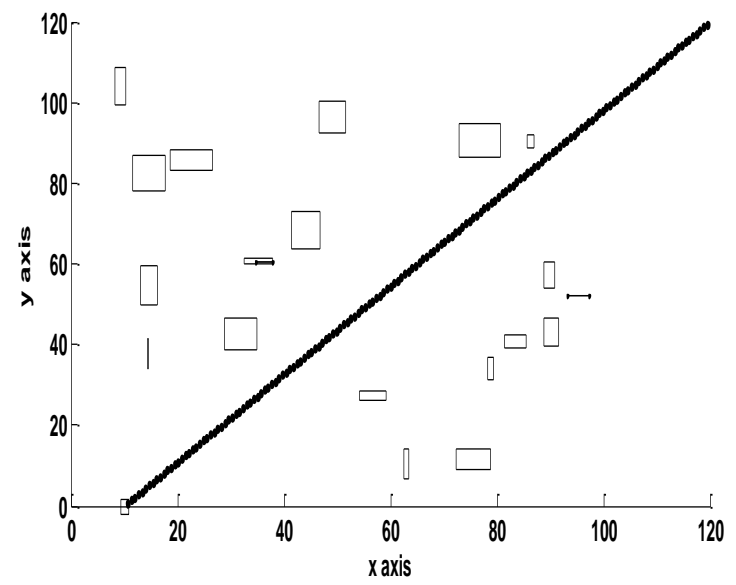

Fig. 4 Robot Reaching Target without Encountering Obstacle

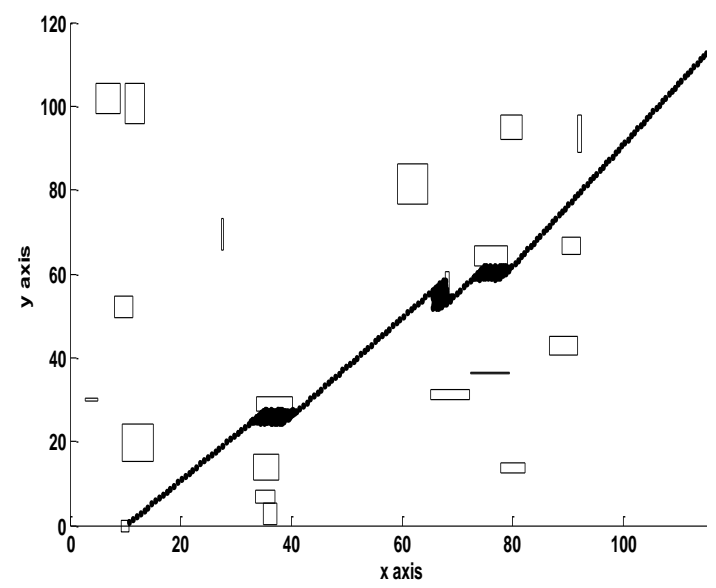

Fig. 5 Robot Reaching Target using BA

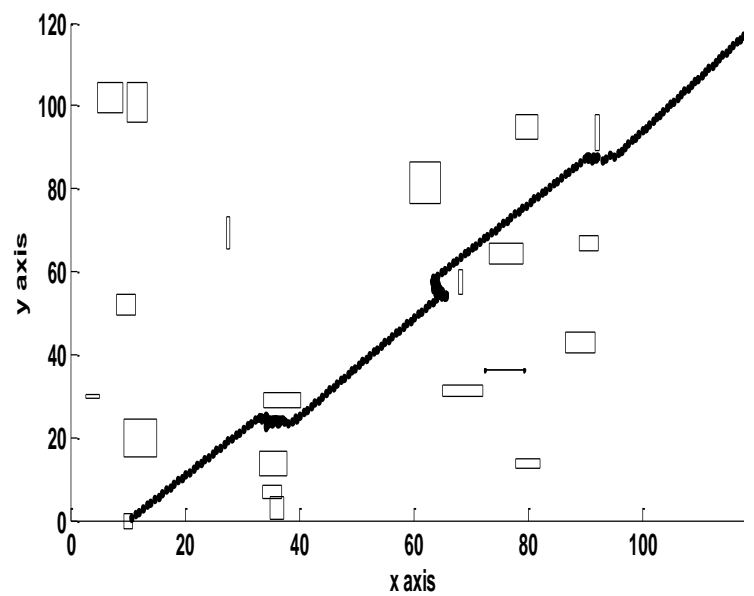

Fig. 6 Robot Reaching Target using EOBA

But if the robot detects an obstacle in the path while moving towards the target,
EOBA was implemented as described in Figure 3 to steer the robot within the environment while ensuring optimal path as shown in Figure 5 and 6.

\section{RESULTS}

After applying standard bat algorithm and elite opposition based bat algorithm for the determination of an optimal path without colliding with obstacles as shown in Figures 5 and 6, it is evident that the path followed by the robot when using EOBA is optimal than that followed using BA. In addition, the results of Figures 5 and 6 on the basis of time elapsed is elaborated in the Table 1 as follows:

\section{TABLE 1: SUMMARY OF TIME ELAPSED BY BA AND EOBA IN THE SIMULATION ENVIRONMENT}

\begin{tabular}{|c|c|c|}
\hline \multirow{2}{*}{ Algorithm } & $\begin{array}{c}\text { Number of } \\
\text { Obstacles }\end{array}$ & $\begin{array}{c}\text { Time Elapsed } \\
\mathbf{1 2 0} * \mathbf{1 2 0}\end{array}$ \\
\hline \multirow{3}{*}{ BA } & 10 & 45.0412 \\
\cline { 2 - 3 } & 20 & 27.8171 \\
\cline { 2 - 3 } & 50 & 61.6376 \\
\hline \multirow{3}{*}{ EOBA } & 10 & 11.0742 \\
\cline { 2 - 3 } & 20 & 17.4909 \\
\cline { 2 - 3 } & 50 & 35.7726 \\
\hline
\end{tabular}

From the given results in Table 1, the BA algorithm produces a fluctuating results with respect to the time elapsed by the robot to reach its destination as compared with that of EOBA. Also, the time taken by the robot in generating an optimal path using BA is more than that taken by the robot using EOBA algorithm. This shows a minimization in the time taken by the robot to reach the target using the EOBA algorithm when compared with the BA algorithm. Hence, EOBA is better technique for obstcle avoidance due to its capability to converge efficiently and improved performance.

\section{CONCLUSION}

An optimal path planning using Elite opposition Based Bat Algorithm for mobile robot have been developed and the 
performance of the EOBA algorithm has been compared with that of the standard bat algorithm by increasing the number of obstacles in a developed simulation environmet with dimension $120 * 120$. The simulation result obtained shows that EOBA generates better quality solutions with better precision accuracy and higher ability of escaping local minimal when compared with the standard BA algorithm. This indicates the superiority of EOBA over BA as it provide an optimal path with minimum elapsed time without colliding with obstacles.

\section{REFERENCES}

[1] Abbas, N.H. and F.M. Ali, Path Planning of an Autonomous Mobile Robot using Directed Artificial Bee Colony Algorithm. International Journal of Computer Applications, 96(11), 2014.

[2] Yun, S.C., V. Ganapathy, and L.O. Chong. Improved genetic algorithms based optimum path planning for mobile robot. in Control Automation Robotics \& Vision (ICARCV), 2010 11th International Conference on. 2010. IEEE.

[3] Raja, P. and S. Pugazhenthi, Optimal path planning of mobile robots: A review. International Journal of Physical Sciences, 7(9): p. 1314-1320, 2012.

[4] Negnevitsky, M., Artificial Intelligence: A Guide to Intelligent Systems. 2002.

[5] Yang, X.-S., A new metaheuristic bat-inspired algorithm, in Nature inspired cooperative strategies for optimization (NICSO 2010), Springer. p. 65-74, 2010.

[6] Alihodzic, A. and M. Tuba. Improved hybridized bat algorithm for global numerical optimization in Computer Modelling and Simulation (UKSim), 2014 UKSim-AMSS 16th International Conference on. 2014. IEEE.

[7] Tizhoosh, H.R. Opposition-Based Learning: A New Scheme for Machine Intelligence in CIMCA/IAWTIC. 2005.

[8] AI, B., M.-G. DONG, and C.-X. JANG, Simple PSO Algorithm with Opposition-based Learning Average Elite Strategy complexity, 9(6), 2016.

[9] Zhou, X., Z. Wu, and H. Wang. Elite opposition-based differential evolution for solving large-scale optimization problems and its implementation on GPU in 2012 13th International Conference on Parallel and Distributed Computing, Applications and Technologies. 2012. IEEE.

[10] Zhou, Y., R. Wang, and Q. Luo, Elite opposition-based flower pollination algorithm. Neurocomputing, 188: p. 294-310, 2016.

[11] Zhao, J. and L. Lv, Shuffled frog-leaping algorithm using elite opposition-based learning. International Journal of Sensor Networks, 16(4): p. 244-251, 2014.

[12] Tijani, S.A. and M. Mua'zu. Stabilization of inverted pendulum system using intelligent Linear Quadratic Regulator controller in Computational Intelligence (IJCCI), 2015 7th International Joint Conference on. 2015. SCITEPRESS. 
Yanbu Journal of Engineering and Science Vol. 16 (2018) 
